\title{
L’Été 80 de Marguerite Duras ou la Normandie dépaysée
}

\author{
Philippe Vilain \\ Université Paris III
}

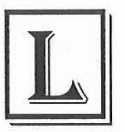

es dix chroniques de Marguerite Duras, publiées dans Libération à la demande de Serge July, directeur du quotidien, avant d'être réunies dans un petit livre marginal et assez méconnu du grand public intitulé L'été 80 , relatent l'histoire d'amour platonique entre une jeune anglaise de dix-huit ans, monitrice de colonie de vacances, et un garçon de six ans; histoire dont l'inceste ici idéalisé, à peine dévoilé, évoque déjà aux durassiens les plus avertis quelques scènes de Barrage contre le Pacifique et préfigure un thème clef, obsessionnel - l'amour pour le petit frère - de l'œuvre, présent notamment dans Agatha ou dans L'amant. La part transgressive de ce texte, qui n'en constitue pas toutefois l'intérêt fondateur, se trouve ici diluée dans " une sorte d'actualité parallèle ${ }^{1}$ " relative au contexte politique mondial très agité qui commence la décennie des années 80 et achève le septennat de Valéry Giscard d'Estaing - les affaires du Parti Communiste de Georges Marchais inféodé à l'Union soviétique, l'inauguration par Brejnev des Jeux Olympiques de Moscou où la participation des sportifs français fut un temps mise en cause, la flambée du pétrodollar koweïtien, l'enterrement du Shah d'Iran et le retour au pouvoir des islamistes de l'ayatollah Khomeiny, l'attentat meurtrier de Bologne attribué aux Brigades rouges, la famine en Ouganda et, notamment, la grève des chantiers navals de Gdansk. Ce sont ces événements que Marguerite Duras fait revivre depuis son appartement de l'hôtel des Roches Noires où elle passe l'été, qu'elle projette sur la plage désertée en raison des épouvantables conditions climatiques en cette période estivale, sur cette petite et calme station balnéaire de Normandie, Trouville, dont un autre deux autres écrivains (Gustave Flaubert transi par le souvenir de son amour de jeunesse Elisa Schlésinger et Marcel Proust qui s'inspire partiellement de Trouville pour décrire Balbec dans $A$ l'ombre des 
jeunes filles en fleurs) avait déjà fait la gloire un siècle plus tôt. Il s'agira ainsi de voir comment Marguerite Duras, enfermée dans son appartement de Trouville, réussit à déplacer les lieux qu'elle décrit, comment elle retire à Trouville sa normandité et dépayse l'espace normand au profit d'un espace universel.

Il peut paraître difficile d'identifier Trouville, station balnéaire annexe de Deauville située sur la côte fleurie entre Honfleur et Cabourg, tant la physionomie, l'histoire et les mours de la ville font si peu l'objet de description. Un décalage très important s'opère entre l'apparent réalisme de la description fondée sur l'identification des lieux de référence (noms de rue), et l'abstraction constante à laquelle sont soumis ces lieux. Trouville s'apparente à un endroit de nulle part, représentation symbolique et typique du lieu de vacances, à forte concentration touristique telle qu'il peut en exister un peu partout dans le monde et dont on trouve déjà le modèle exemplaire, palimpseste, dans les premiers romans durassiens : Trouville semble être en effet une réplique du village décrit dans Les petits chevaux de Tarquinia, dans Le Marin de Gibraltar ou dans L'Amour. Répétés, presque identiques dans leur géométrie, cernés eux aussi par la mer, ces petits ports de pêche se superposent de texte en texte pour provoquer un sentiment d'incertitude du réel. Tout se passe, en effet, comme si la station de Trouville que les chroniques tentent pourtant de décrire au moyen de référents réalistes, subissait une sorte de brouillage topographique. Trouville, le plus souvent désignée de façon impersonnelle (" la ville »), est mise en évidence de façon presque exclusive par l'omniprésence de la pluie, de la tempête ou de la brume d'une "épaisseur insondable ", des éléments et variations climatiques qui empêchent toute tentative de repérage et plonge de fait ces lieux authentiques dans un brouillard procédant lui-même d'un imaginaire de la réalité ${ }^{1}$. L'espace réaliste se trouve déréalisé au profit d'un espace parfaitement nivelé, sans profondeur, sans perspective, extensif, répandu sur la platitude infinie de la terre qui contamine la surface et l'horizon de la mer :

La permanence des mêmes lieux, la fidélité quasi physique de Marguerite Duras aux espaces sableux, à la chaleur, à la mer surtout, les rendent fictifs. La réalité géographique de Calcutta, Lahore, S. Thala, importe peu; certaines de ces villes existent sur les cartes, d'autres non; mais l'essentiel, c'est que l'histoire soit ailleurs, piégée dans l'univers durassien ${ }^{2}$. 
S'interdisant toute limitation, la ville permet l'abolition des frontières. L'écriture semble produire un écartèlement spatial en juxtaposant le monde terrestre au monde marin. La description des surfaces et des lignes, des ombres et des lumières, est en même temps véhiculée par un langage géométrique dont l'abstraction et l'extrême précision, loin de montrer les choses, aplanissent ce même lieu pour lui donner une configuration uniforme. L'analogie avec la peinture abstraite de Nicolas de Staël, à laquelle Marguerite Duras se réfere pour décrire la ville normande de Quillebeuf sur Seine ${ }^{3}$, est évidemment tentante : la même épuration spatiale, la même valorisation de la surface déserte et dépeuplée au profit des lignes, la même absence de l'humain dans le paysage pictural entrent dans l'écriture durassienne.

Cette vision de l'espace, et plus généralement du réel, coupé de ses relais avec l'homme, aux antipodes d'une vision de l'espace romantique prêtant à la nature expression et sensibilité, pourrait être rapprochée de la vision proustienne, cherchant à capter l'essence des choses. Les endroits, souvent évoqués de façon allusive à partir d'une perception visuelle ou d'une perception auditive, ne visent pas seulement à donner une représentation désubstantialisée du réel, mais participent surtout d'une représentation du temps durassien. Ici, la traversée de l'espace se métaphorise toujours en effet en une traversée du temps :

On marche, on écrit, on traverse la ville, elle est traversée, elle cesse, la marche continue, de même on traverse le temps, une date, une journée et puis elle est traversée, cesse $e^{4}$.

L'été 80 permet donc l'absolue coïncidence du temps et de l'espace, une forme de syncrétisme spatio-temporel qui passe et se réalise par le regard, véritable instrument de ravissement du monde. Lêtre durassien se borne le plus souvent au constat de ce qu'il voit ou croit voir, sans donner d'interprétation, sans déchiffrer le réel. Par le regard, sa conscience tend ainsi à se fondre dans l'univers même. L'enfant aux "yeux incommensurablement gris » devient une simple zone de transfert opérant lui-même une continuité de l'espace :

Ses yeux sont gris comme l'orage, la pierre, la mer, l'intelligence immanente de la matière, de la vie. Gris, les yeux couleur du gris, comme une teinte extérieure posée sur la force fabuleuse de leur regard 5 . 
Le regard englobe le monde et réciproquement, de sorte que l'être durassien, prisonnier des pluies de Trouville, semble s'abandonner à une rêverie doublée d'un projet de fuite. Le besoin d'étendue est logé au cour de la perception de l'être. Siège de la captation et de l'expulsion, le regard évoque la forme du miroir : il reçoit le monde de façon à l'écouler vers le dehors qui, dans un mouvement inverse, comble à son tour l'espace vide du dedans. L'être durassien traverse l'espace en même temps qu'il se trouve traversé par l'espace. Son regard-au-monde capte tout le champ de l'existence au point de rendre inopérante la dichotomie intérieur/extérieur. Nous avons même le sentiment que l'être durassien se chosifie pour humaniser la matière. Ce mimétisme - ou tout simplement vision concrète de l'essence des choses - est suppléé par la perspective narrative qui marque elle-même une continuité spatiale intertextuelle.

La narratrice, cloîtrée dans sa chambre de Trouville, parcourt le monde d'escale en escale : Moscou, Gdansk, Pékin, l'Afganisthan, Southampton, Glasgow, Edimbourg, Dublin, Lille, Brest, Cabourg, Munich, Melbourne, Vancouver, Paris... Ces voyages illustrent la recherche non d'une voie mais d'une issue que ne cesse de se dérober vers un autre lieu. La narratrice voyage à l'instinct, comme le faisait l'héroïne du Marin de Gibraltar, sans plan ni boussole. D'une part, elle apparaît porteuse d'une histoire sans lien avec le présent, complètement rattachée au passé. Ses voyages désigneraient le cheminement d'un inconscient vers ce centre opaque, originel, vers ce vide qui le constitue, à son insu, et dont l'image mythique serait la présence immuable de la mer, ressouvenir sans doute de l'enfance passée dans l'océan Pacifique près du Mékong. Comme dans Barrage contre le Pacifique, la mer déclenche le mouvement rétrospectif de la mémoire, le retour sur soi. La pénétration dans le monde des souvenirs continue ici une sorte d'espace autobiographique. D'autre part, son parcours reste celui de l'instance productrice du texte, qui entreprend, avec ce livre, un voyage dans le temps. L'espace réel devient ainsi le lieu privilégié de l'errance imaginaire et intérieure, d'un vagabondage mental où resurgissent tour à tour les bateaux de pêche, les grands pétroliers d'Antifer, " le bruit des moteurs et celui de l'eau rumuée, les voix et les appels des pêcheurs du Gange $^{6}$ " qui peuplent les lieux familiers de la Durasie et assurent tout à la fois dans L'été 80, le fictionnement et le dépaysement du paysage normand. 


\section{Notes}

* Centre d'Etudes du Roman du second demi-siècle. Marguerite Duras, L'été 80, Paris, Editions de Minuit, 1980, p. 7.

1 Lire à ce propos l'étude d'Yvonne Guers-Villate, "L'Imaginaire et son efficacité chez Marguerite Duras ", in Les Lettres Romanes, XXIX, 1975, p. 207-217.

2 Gisèle Bremondy, "La destruction de la réalité ", in L'ARC (Marguerite Duras), $n^{\circ} 98,1985$, p. 51.

3 Marguerite Duras, Emily L., Paris : Ed. de Minuit, 1987. p. 63.

4 L'été 80, op. cit., p. 9.

5 Ibid., p. 16.

6 Ibid., p. 35. 